\title{
Characterization of Vacuum Facility Background Gas Through Simulation and Considerations for Electric Propulsion Ground Testing
}

\author{
John T. Yim ${ }^{1}$ \\ NASA Glenn Research Center, Cleveland, OH, 44135 \\ Jonathan M. Burt ${ }^{2}$ \\ U.S. Air Force Research Laboratory, Wright-Patterson Air Force Base, OH, 45433
}

\begin{abstract}
The background gas in a vacuum facility for electric propulsion ground testing is examined in detail through a series of cold flow simulations using a direct simulation Monte Carlo (DSMC) code. The focus here is on the background gas itself, its structure and characteristics, rather than assessing its interaction and impact on thruster operation. The background gas, which is often incorrectly characterized as uniform, is found to have a notable velocity within a test facility. The gas velocity has an impact on the proper measurement of pressure and the calculation of ingestion flux to a thruster. There are also considerations for best practices for tests that involve the introduction of supplemental gas flows to artificially increase the background pressure. All of these effects need to be accounted for to properly characterize the operation of electric propulsion thrusters across different ground test vacuum facilities.
\end{abstract}

\section{Nomenclature}

$k=$ Boltzmann constant

$m=$ Molecular mass

$n$ = Number density

$p$ = Pressure

$T$ = Temperature

$v_{B}=$ Bulk velocity

$v_{t h}=$ Thermal velocity

\section{Introduction}

$\mathrm{T}$

HE effects of finite background gas pressure in ground vacuum test facilities have been of interest in order to properly characterize the operation, performance, and life of electric propulsion (EP) thrusters - especially of late for Hall thrusters. ${ }^{1-10}$ The full mechanisms of facility effects are still not completely understood, and contributing to the confusion is the variability in vacuum facility configurations and facility pressure measurement methods. ${ }^{11}$ These variances can lead to an improper understanding of facility effects which can affect the qualification, acceptance, and margins applied to thrusters for integration onto potential missions, and ultimately ease of adoption of EP by the spacecraft community. As NASA and others show interest in thrusters at a higher power level than current state of the art, such as the $12.5 \mathrm{~kW}$ HERMeS Hall thruster under development, ${ }^{12}$ these potential issues can become more salient as higher demands are pushed on existing facility capabilities.

The work presented here focuses on the nature of the background gas itself. Often it is easy to assume that the background gas in a vacuum facility is some uniform and static presence, simply and adequately described by a single pressure value. However, the structure of the background gas flowfield may not be so simple, and care should be taken to assess its properties and potential subsequent consequences on thruster operation and performance. A series of cold flow simulations of vacuum facility operation were run and analyzed to explore the nature of the

\footnotetext{
${ }^{1}$ Aerospace Engineer, Electric Propulsion Systems Branch.

${ }^{2}$ Senior Researcher, Ohio Aerospace Institute.
} 
background gas and to examine what potential lessons may be discovered from even these simple studies. The main findings of this work show that the facility background gas is neither static nor uniform. The presence of a bulk velocity for the background gas flow has implications that affect calculating the flux at the thruster, making facility pressure measurements, and artificially increasing the background pressure through additional gas flow. Assessments of the magnitude of these impacts are provided, though it should be noted that these results will be heavily dependent on the particular thruster and facility configuration under consideration.

\section{Model Description}

Simulations of vacuum facility background gas were performed using the Hypersonic Aerothermodynamics Particle (HAP) code, a general Cartesian implementation of Direct Simulation Monte Carlo (DSMC), with features including shared memory parallelization and the capability to automatically generate triangulated surfaces based on analytical geometry definitions. ${ }^{13}$ DSMC is well suited to model the rarefied flow within vacuum chambers and has been used to analyze other vacuum facilities. ${ }^{14}$ For the purposes of this paper, Vacuum Facility 5 (VF-5) at NASA Glenn Research Center is used as a representative test facility to highlight certain findings. The details of the VF-5 model including correlations to measured test data are described in another report. ${ }^{15}$

It should be clearly noted that the simulation results presented in this work are solely of cold flow gas. The gas inflow is modeled as a diffuse inflow of neutral gas particles typically at room temperature. No ions, no electromagnetic fields, no charge exchange collisions, and no accelerated particles simulating high exit velocities are simulated. The neutral flux profile is modeled as a diffuse flow through an annulus, but during Hall thruster hot fire operation, the neutral density profile can have a very non-uniform profile with distinct peaks near the walls of the discharge channel and a neutral density lower than that of the background gas at the channel centerline. ${ }^{16}$ However, in spite of the lack of these physics, the overall pressure profile in the vacuum facility can still be fairly well captured. ${ }^{15,17}$ Obviously a full treatment of facility effects should include a detailed plume model with the appropriate physics. However, for the purposes of this work - which focuses more on the nature of the background gas itself, rather than on its interaction with the thruster and plume - even these simple cold gas models can provide some useful insight.

To help isolate the effects of the background gas itself apart from the expanding plume flow, the DSMC simulation particles are tagged either as "plume" particles or "background" particles. Particles introduced into the simulation domain through the thruster are tagged as plume particles. Once a plume particle impacts a chamber surface, it is converted into a background particle. All of the physical and simulated characteristics remain the same, just the label attached to the particle is switched. If a plume particle collides with a background particle, it is also converted into a background particle; however, if two plume particles collide together, they both remain as plume particles. This particle characterization helps to identify the gas flow properties that are affected by the presence of a facility, and what properties remain if the inflow were to freely expand in space.

\section{Results and Discussion}

\section{A. Background gas characteristics}

Model results of a sample ground test configuration are shown in Figure 1, where a slice of the VF-5 geometry along the thruster centerline is shown including two cryopumps within that plane depicted by the white rectangles. The location of the thruster is depicted by the small cylinder symbol, and the thruster is pointing to the right. The overall number density profile in the chamber is provided in the upper left part of the Figure 1. The lower left part of Figure 1 provides the number density profile of just the background gas. The overall density profile is rather similar between the two, particularly upstream of the thruster and far downstream. The plume gas is only in significant quantity in the near field region, which is seen in the top right portion of Figure 1. For comparison purposes, a simulation was run simulating the expansion of the inflow in free space, shown in the bottom right. For that case, all of the particles are "plume" since there is no background facility to interact with. The profiles between the plume gas only and the free expansion in open vacuum are very similar to each other. The only apparent difference is the lower density of the chamber plume gas as it is depleted with interactions of the chamber and background gas. 

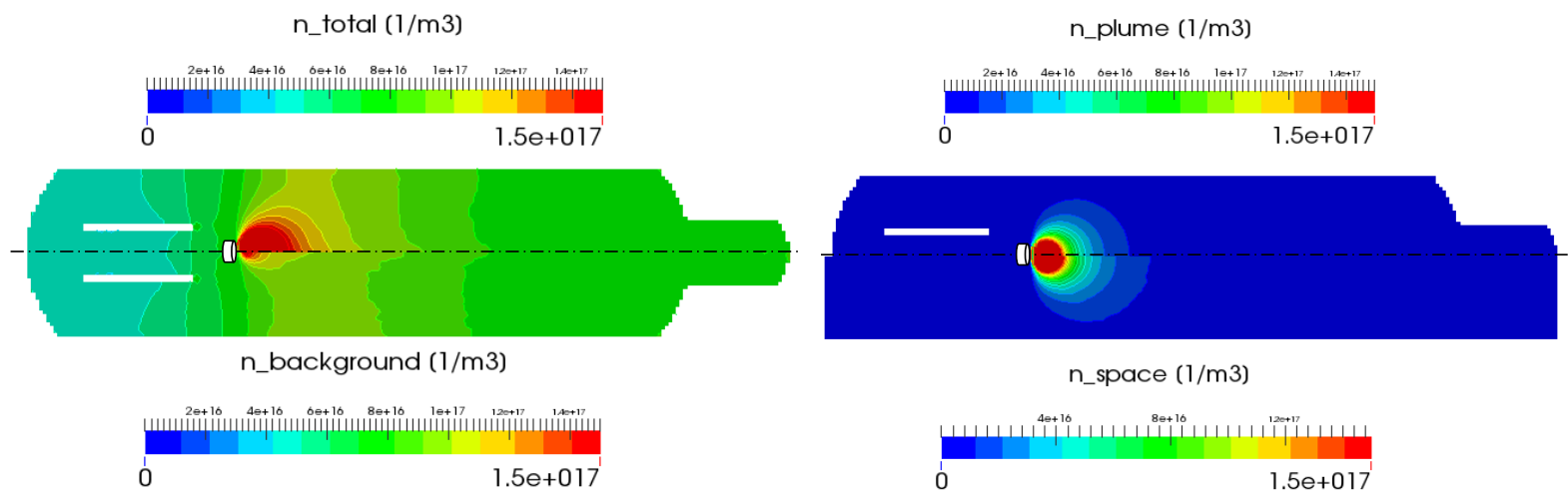

Figure 1: The simulated number density in the chamber for total gas (top left), background gas only (bottom left), plume gas only (top right), and total gas in free space (bottom right).

A more detailed examination of the density profile radially along the thruster exit plane is shown in Figure 2. Here, the total number density and the component densities of the plume and background gas are shown. It is seen that the plume flow dominates close to the thruster exit, but further away from the inflow-the outer radius of the inflow annulus for these models is approximately $0.1 \mathrm{~m}$ - the flow is essentially just the background gas. In addition, the radial profile of the background gas density does not change significantly as it crosses in front of the thruster. A slight increase is seen and this is directly related to the high density of plume gas which is converted to background gas through collisions, rather than just background gas migrating to the thruster exit plane from elsewhere in the facility. Please note this behavior seen here for cold diffuse flow is markedly different than a hot fire condition with a discharge plasma plume, where charge exchange collisions will deplete the neutral population in front of the thruster due to fast neutrals. ${ }^{6}$ However, in this study, we would like to consider just the population of incoming flux of background gas particles before they encounter "plume" particles, whether a cold gas or a hot plasma; this will be further described and explored below.

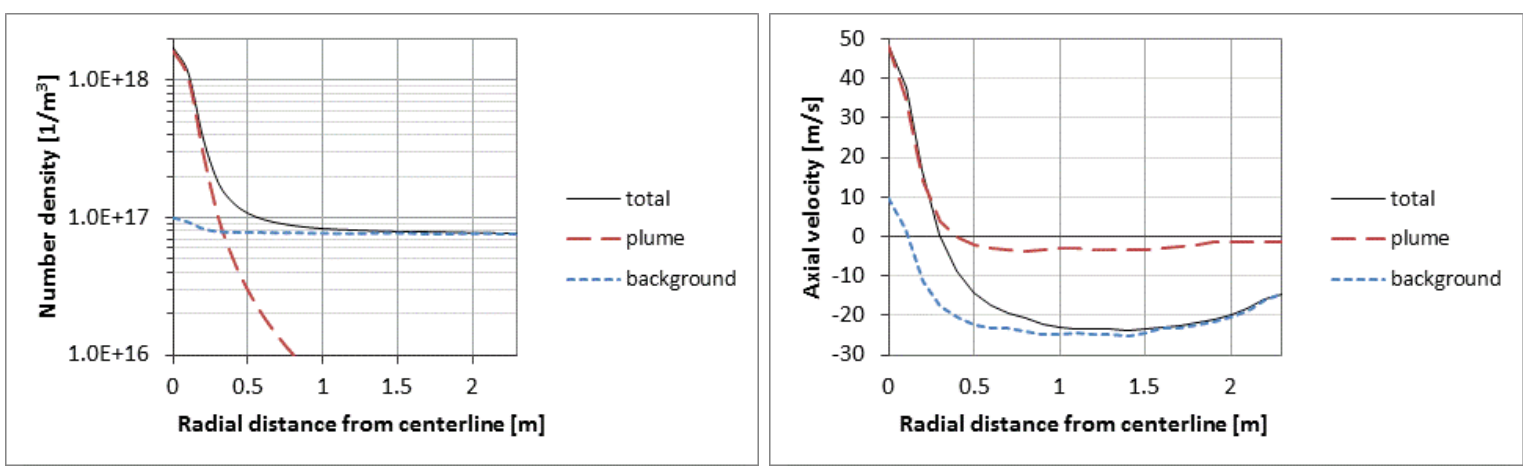

Figure 2: The radial profiles of number density (left) and axial velocity (right).

Much emphasis in facility characterization for EP ground testing is focused mainly on the background pressure as it is the most readily measured. However, it is yet unclear what parameters of the background gas play influential roles in interacting with thrusters and their plumes. A bulk velocity present in the background gas will affect the background gas number flux to the thruster. The axial velocity radially along the thruster exit plane from the model is shown on the right side of Figure 2. Directly in front of the thruster, there is a clear velocity in the "plume" flow, which is also noticeable in the background flow as it is entrained by the expanding plume. Outside of the inflow source, however, the flow reverses direction and heads upstream of the thruster. This general flow at the exit plane that proceeds upstream is to be expected as some of the gas from the thruster and rest of the chamber will flow towards the pumps located behind the thruster. The presence of this bulk velocity of the background gas has some implications that will be further examined herein. 


\section{B. Implications for facility pressure measurements}

Now consider the axial variations in pressure at a given radial location. The plot to the left in Figure 3 graphs the normalized total, plume, and background density axial profiles at a radial location approximately halfway between the thruster and facility wall. The first observation is that the plume gas is essentially negligible upstream of the thruster, but is present downstream. Focusing more on the upstream side of the thruster, where the density is essentially just that of the background gas, the density is seen to drop with increasing distance from the thruster. It is approximately $10 \%$ lower $50 \mathrm{~cm}$ behind the thruster. The right side of Figure 3 shows the axial profile of the bulk velocity of the background gas at four radial locations, wall is near the facility wall, mid is approximately halfway between the facility wall and the thruster, near is outside of the thruster inflow approximately two thruster diameters from the centerline, and thruster is within the inflow outer radius. Clear entrainment of the background gas is seen in front and near the thruster, but negligible further away, and this entrainment is primarily downstream of the thruster. These results suggest measurement of the facility pressure should be taken at or perhaps slightly upstream of the thruster exit plane to most accurately characterize the properties of just the background gas at the exit plane.

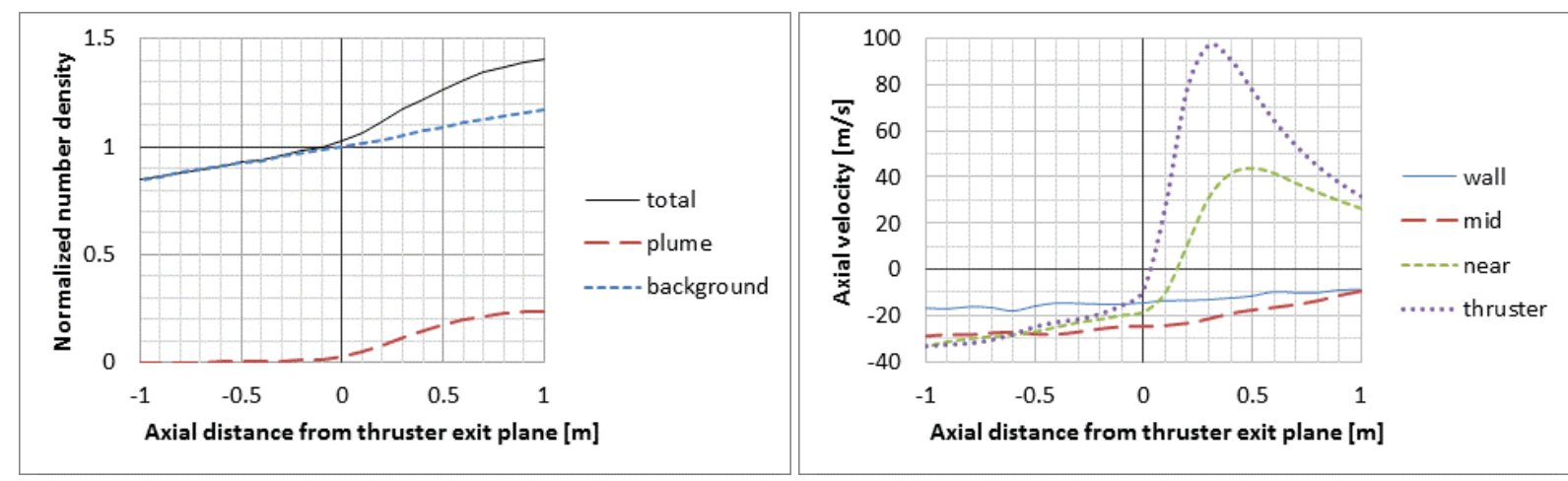

Figure 3: Axial profiles of normalized density at the mid radial location (left) and background gas bulk axial velocity at four radial locations (right).

For thruster ingestion studies, it would perhaps also be useful to identify the flux of background gas arriving from the chamber versus the generation of "background" gas arising from collision interactions with the plume in the near field region as noted above. To help assess this issue, velocity distribution functions (VDF) were generated from the DSMC code at several locations. Four VDFs from the four radial locations along the thruster exit plane previously described above are shown on the left hand side of Figure 4. There is a clear peak for the near and thruster curves arising from the inflow. If we look just at the thruster location and split up the VDF into contributions from the inflow plume source and the background gas, the positive velocity region is dominated by the plume gas, while the contributions of the background gas lie towards the negative end. Interestingly, the background gas VDF is comparable to the total VDFs at the mid and wall locations, indicating the background inflow is similar to the flow further out radially. This suggests measurement of the facility pressure radially away from the thruster along the exit plane will provide a fair estimate of the flux of background gas at the thruster itself. This has also been seen in other experiments and simulation. ${ }^{9}$ Caution should be taken, though, before overgeneralizing this result; different facility and thruster configurations could lead to different results.

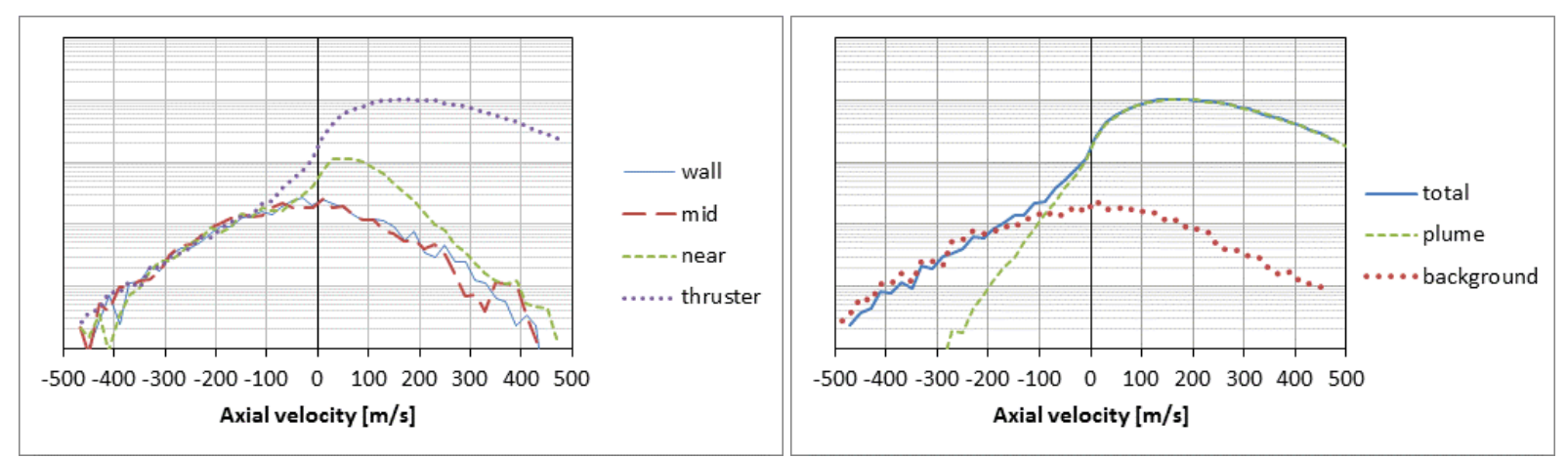

Figure 4: The total (background and plume) velocity distribution functions along the thruster exit plane at different radial locations (left) and the velocity distribution function contributions at the thruster exit location only (right). 
The presence of a background gas velocity within the facility also has some implications for facility pressure measurement. Typically, the recommended setup for an ion gauge for EP applications includes the use of a neutralizer tube attached to the gauge inlet as shown in Figure $5 .^{11}$ This tube is used to protect the gauge from direct contact with the plume plasma, which can interfere with proper measurement of facility pressure. However, depending on the direction of the neutralizer inlet relative to the local background gas flow direction, the pressure in the neutralizer and measured by the gauge can be different than the pressure outside of the neutralizer inlet. The use of the neutralizer tube creates a region of stagnated flow within the tube and the stagnation pressure can be different from the local static pressure at the entrance of the neutralizer.

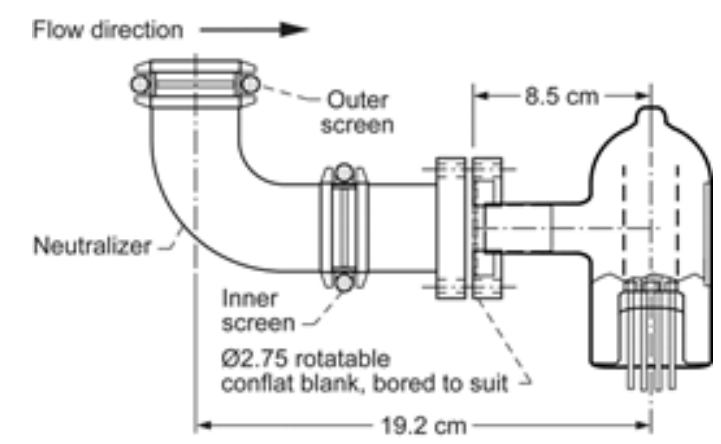

Figure 5: The recommended ion gauge and neutralizer setup from Dankanich, et al. ${ }^{11}$

From an analytical perspective, the equations for stagnation pressure for continuum flow are well known. For a Maxwellian free molecular flow, a relation for an infinitely long tube at a zero angle of attack relative to the flow has been derived ${ }^{18}$

$$
p_{\text {tube }}=p_{\infty} \sqrt{\frac{T_{\text {wall }}}{T_{\infty}}}\left\{\left[\frac{S}{\sqrt{\pi}}+\frac{1}{2}\right]\left[e^{-S^{2}}+\sqrt{\pi} S(1+\operatorname{erf}(S))\right]+\frac{1}{2}[1+\operatorname{erf}(S)]\right\} ; \quad S=\frac{v_{B}}{\sqrt{\frac{2 k T_{\infty}}{m}}}
$$

where $T_{\text {wall }}$ is the temperature of the tube walls and $S$ is the ratio of the bulk velocity, $v_{B}$, to the most probable molecular speed. Relations for tubes of arbitrary length also exist, ${ }^{19}$ and though no closed form analytical solutions are available for this more general case, numerical solutions can be calculated. Note that these relations also assume the tube is oriented into the flow with zero angle of attack. If the gauge is located in a transitional region between continuum and free molecular flow, curve fits have also been found bridging this regime. ${ }^{20}$

A set of DSMC simulations were run incorporating a representative neutralizer tube within a Maxwellian equilibrium rarefied background gas flow. Sample results are shown in Figure 6 with the neutralizer tube represented as a cylinder open at one end for simplicity, with three orientations - facing into, sideways, and away relative to the background gas flow-were modeled. The neutralizer tube facing into the flow is seen to experience a sense volume with elevated pressure relative to the surrounding flow. The DSMC-calculated relative pressure for this case is seen to correspond well to the theoretical values as shown in Figure 7. Conversely, a tube oriented away from the direction of flow is seen to experience a lower pressure that is of the same relative, but opposite, magnitude as the forward facing case. This has also been seen experimentally during HiVHAc thruster testing in VF-5 where a gauge facing upstream relative to the thruster, but away from the direction of background gas flow, recorded pressures that were found to be $28-37 \%$ lower than corresponding pressures measured from the downstream facing gauge. ${ }^{7}$ The case of a tube inlet pointed perpendicular to the direction of flow, as shown in the middle plot of Figure 6 , does not show any significant pressure deviations from the surrounding gas. Similar simulations were performed for elbow and tee tube configurations, and they produced results similar to the straight tube cases shown below in Figure 6. One other observation is that it is seen that neutralizer tubes with a length to diameter ratio over unity can for practical purposes be approximated by the solution for an infinite length tube, given above in Eq. (1), for low flow rates. 

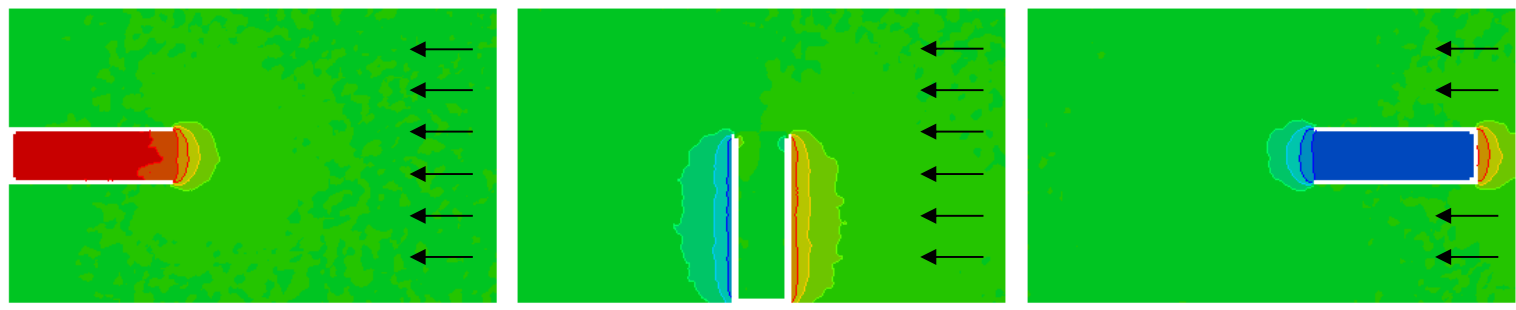

$P$ (normalized)

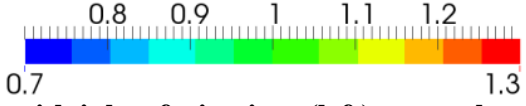

Figure 6: Pressure ratios for closed tubes with inlets facing into (left), normal to (middle), and away from the flow (right).
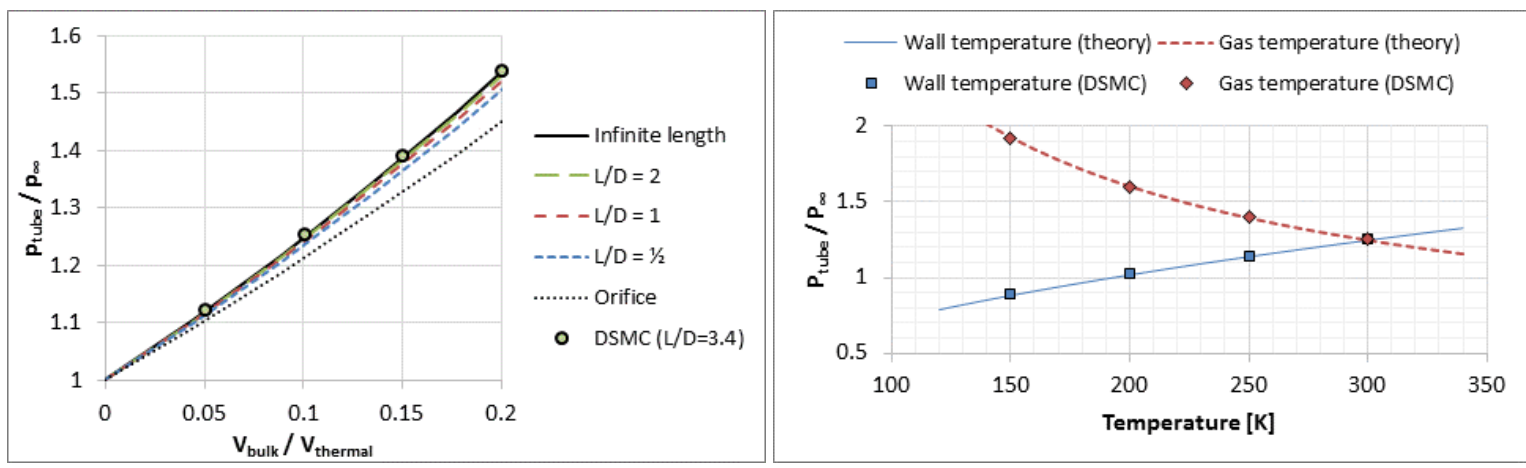

Figure 7: The ratio of pressure in a neutralizer tube to the freestream free molecular flow for tubes of various length to diameter ratios (left) and the effect of different tube wall and gas temperatures (right).

In addition to the bulk velocity of the flow, the pressure inside of the neutralizer tube, and sensed by the ion gauge, is dependent on the temperatures of the tube wall and the inflow gas. ${ }^{21,22}$ Ion gauges being cooled by nearby cryosurfaces, or perhaps heated by the thruster or the gauge itself, can have temperatures deviating from room temperature, while the facility background gas can also be at varying temperatures as discussed above. Thus, a proper measurement of the facility pressure should include temperature corrections. The plot on the right hand side of Figure 7 shows the resulting pressure in the tube is proportional to the square root of the tube wall temperatures when the gas temperature is the same (here at $300 \mathrm{~K}$ ). The bulk velocity and the length to diameter ratio of the tube are held constant for these results. The effect of the surrounding gas temperature is more complex as it also affects both the most probable and thermal speeds of the gas. This trend is also shown on the right side of Figure 7 which assumes a constant $300 \mathrm{~K}$ tube wall temperature. A ion gauge with neutralizer tube walls cooler than the gas being measured will sense a gas pressure lower than the true pressure outside of the sense volume. A proper correlation of the measured pressure to the true freestream pressure should then incorporate effects from both flow anisotropy and gas and wall temperatures.

The relation between the stagnated flow pressure and the surrounding freestream pressure can also be used in reverse to estimate the static pressure and flow velocity from stagnation pressure measurements. Two ion gauges can be placed such that the neutralizers are oriented both into and away from the background gas flow direction, and such that they are in a similar region of flow that the properties of the background gas can be expected to be similar. Then, assuming the local pressure is the same and the flow velocity is similar but opposite direction, the ratio of the two stagnation pressures can be used with Eq. (1) to back out the estimated velocity, as shown on the right side of Figure 7, and subsequently the associated static pressure. As an example, these calculations were carried out on HiVHAc pressure data which included both upstream and downstream facing gauges. ${ }^{7,8}$ Making the assumption the background gas was flowing in the axial direction in the facility at the location of the gauges, the resulting static pressure and background gas velocity are then calculated with results shown in Figure 8 for changing the inflow mass flow rate at both the thruster and the supplemental gas flow introduced to increase the backpressure. The static pressure is found to lie in between the two measured values, just below their arithmetic average. The magnitude of the bulk velocity does not appear to show any clear trends with increasing thruster mass flow rate, but remained within $13-17 \mathrm{~m} / \mathrm{s}$ as shown on the left plot of Figure 8 . However, it should be noted that the velocity calculated in this manner is sensitive to the input pressure readings. For example, if the uncertainty is $\pm 6 \%$, as is reported for these pressure measurements, the corresponding uncertainty in the velocity can be up to $\pm 30 \%$. Simulation results 
for this test setup show that the velocity does not show any significant changes or trends with increasing flow rate at the thruster, though the noise in the results may obscure any minor trends. When a supplemental gas inflow downstream of the thruster is increased, however, a corresponding increase is seen in the background gas velocity as shown on the right side of Figure 8. Simulation results also reflect this trend.
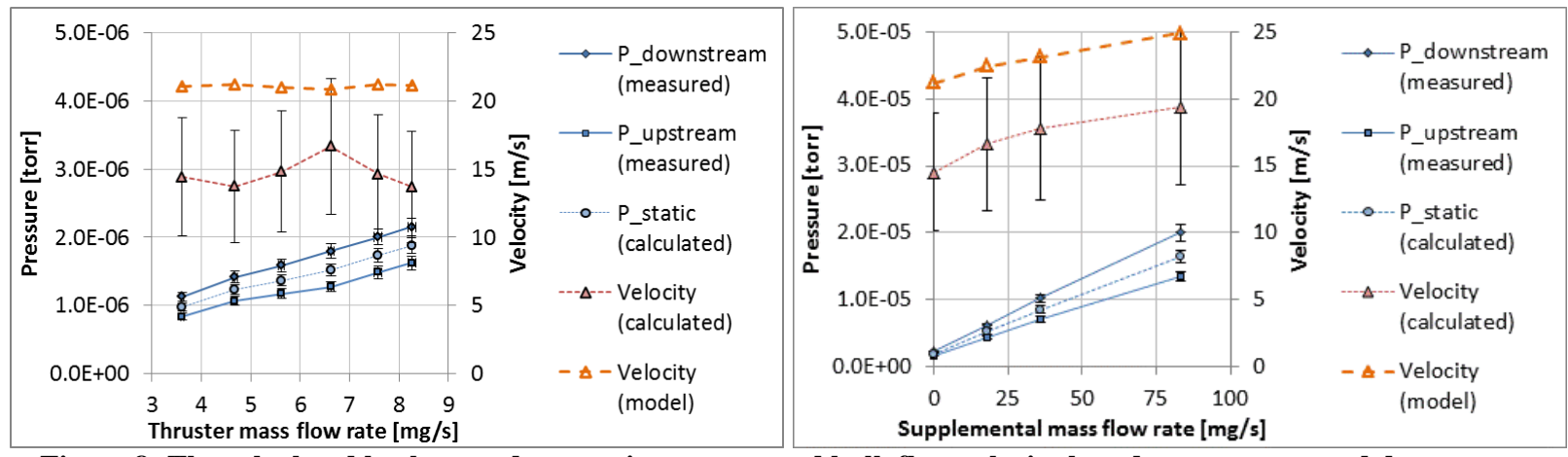

Figure 8: The calculated background gas static pressure and bulk flow velocity based on upstream and downstream facing ion gauge pressure measurements during HiVHAc thruster testing versus thruster mass flow rate (left) and supplemental gas flow rate (right).

A similar approach to estimating the background gas velocity can also be performed with one gauge facing into the flow and another gauge perpendicular, or lateral, to the flow, where the latter gauge would have a fair reading of the static pressure at the gauge location. This setup was implemented for preliminary testing of the $12.5 \mathrm{~kW}$ HERMeS thruster. ${ }^{23}$ Three gauges were installed near the thruster, one facing downstream and the other two oriented laterally. The two gauges aligned sideways were in very close agreement to each other, while the one pointed downstream consistently provided a higher pressure reading output as shown in Figure 9. One notable difference between these HERMeS thruster case results and the HiVHAc case above is that the model predicted background gas velocities are consistently higher as compared to test calculations for HiVHAc, but consistently lower for the HERMeS case. It should be noted that the test configurations, that is the position and orientation of the thruster relative to the facility pumps, were considerably different between the two thruster setups where these data were obtained. This also perhaps shows the limitations of the thruster cold flow assumption in the DSMC simulations as these pressure gauges were located relatively near the thruster. That location in the simulations was near the edge of the diffuse cold "plume" particles flow within a region of high velocity gradients that might not be present for a hot fire case. Small shifts in the measurement location can affect the end results greatly. In general, though, the general magnitude of the background gas bulk velocity is captured by the model. It is possible the decreasing velocity trend could also be due to potential side effects of the cold flow assumption. The supplemental flow results shown on the right side of Figure 9. Data from two different days of testing are presented and the shift in velocity between the two sets highlight the sensitivity of the velocity calculations to small pressure differences.

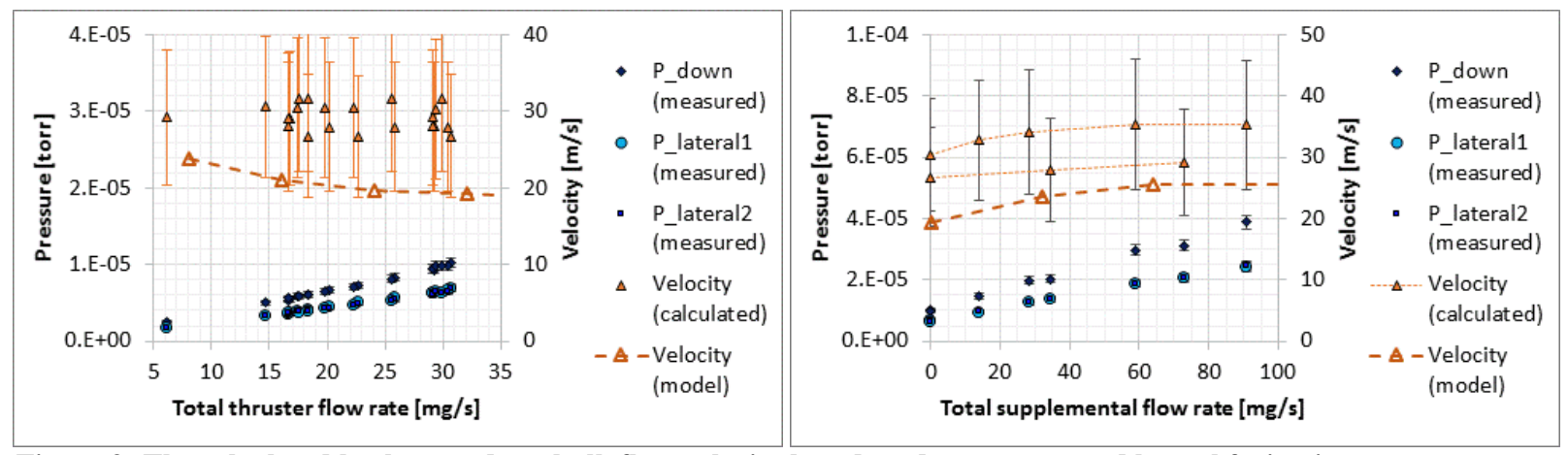

Figure 9: The calculated background gas bulk flow velocity based on downstream and lateral facing ion gauge pressure measurements during HERMeS thruster testing vs thruster mass flow rate (left) and supplemental gas flow rate (right). 
The general recommended orientation for an ion gauge neutralizer tube for best pressure measurement is for the entrance plane to be parallel to the local direction of flow, as shown in Figure 5 and the middle plot of Figure 6, but not facing the thruster as it may then experience direct flux from the thruster plume. Facing the gauge radially away from the thruster, or facing it in an azimuthal or lateral orientation, should not significantly affect the measured pressure from the local flow pressure. Care should be taken not to point the tube entrance towards a pumping surface or other feature that would affect flow direction or the local gas density. The gauge should be placed clear of any other structure or objects that may stagnate the flow and increase the local pressure in front of the tube inlet. Finally, the gauge tube inlet should not be placed close to a wall or other surfaces and create a confined flow volume that may induce conductance losses and perturb the local flow. A second gauge oriented either into or out of the general expected direction of flow can be used to help assess the local background flow bulk velocity which is needed to properly characterize the flux as described earlier above.

\section{Implications for artificially increasing the background pressure}

To characterize thruster operation against different background pressures, typically either pumps are selectively turned off or additional neutral gas flow is injected. The latter method is generally preferred as it provides greater control on the background pressure level. Care must be taken to position and orient the supplementary gas inflow so as to not introduce additional impacts that can unduly affect thruster operation. The desire is for the auxiliary gas flow to not overly perturb the properties of the facility background gas. The flow should also nominally be as uniform as possible across the thruster ingestion area.

A series of simulations were run to examine the effects of different auxiliary inflow configurations. Three axial locations were assessed each with two orientations, one pointed radially towards the facility walls and one pointed axially away from the thruster. Each of the cases were run with the same flow rates. The number density profiles of the background gas across the entire chamber are shown in Figure 10 for the six different cases. The radial profiles at the thruster exit plane for these different cases are shown in the left plot of Figure 11. The density appears to be fairly constant radially for each of the cases with no obvious perturbances in the radial profile shape due to the supplemental gas inflow. The differences in the density increase relative to the baseline case appear to be minor among the cases examined, showing little change due to the supplemental inflow location and orientation. However, the plot on the right side of Figure 11 shows the axial velocity profiles, and here a clear difference is seen for the cases where the auxiliary inflow is behind the thruster as compared to the others; the background gas actually flows in the opposite direction for these setups. The cases where the supplemental inflow is downstream provide a background gas velocity profile comparable to the baseline case. It should be noted that the results presented above on the right hand side of Figure 8 do show a change in velocity with increasing flow rate, so the bulk velocity of the background gas may not necessarily be of the same magnitude as the baseline no additional flow case. Finally, as for radial versus axial supplemental flow injection direction, there are no considerable differences observed here.

As for how far downstream axially the supplemental inflow needs to be, the main criterion would be to minimize any flow non-uniformity seen at the thruster due to a finite inflow location. From the results, there does not appear to be any significant background gas flow radial asymmetries introduced at the thruster. An additional consideration would be to minimize any direct influence of the supplemental gas with the thruster or ion gauge readings. Figure 12 plots the density contours of the supplemental gas that did not interact with the background gas. Note the $\log$ scale on the contour bands indicates the density level at the thruster are several orders of magnitude lower than the background density levels shown earlier. This indicates that either downstream axial location shown here is likely sufficient. Again, the same caveat applies here where these results may not generalize across all thruster and facility configurations, so care should be taken when applying these results. 


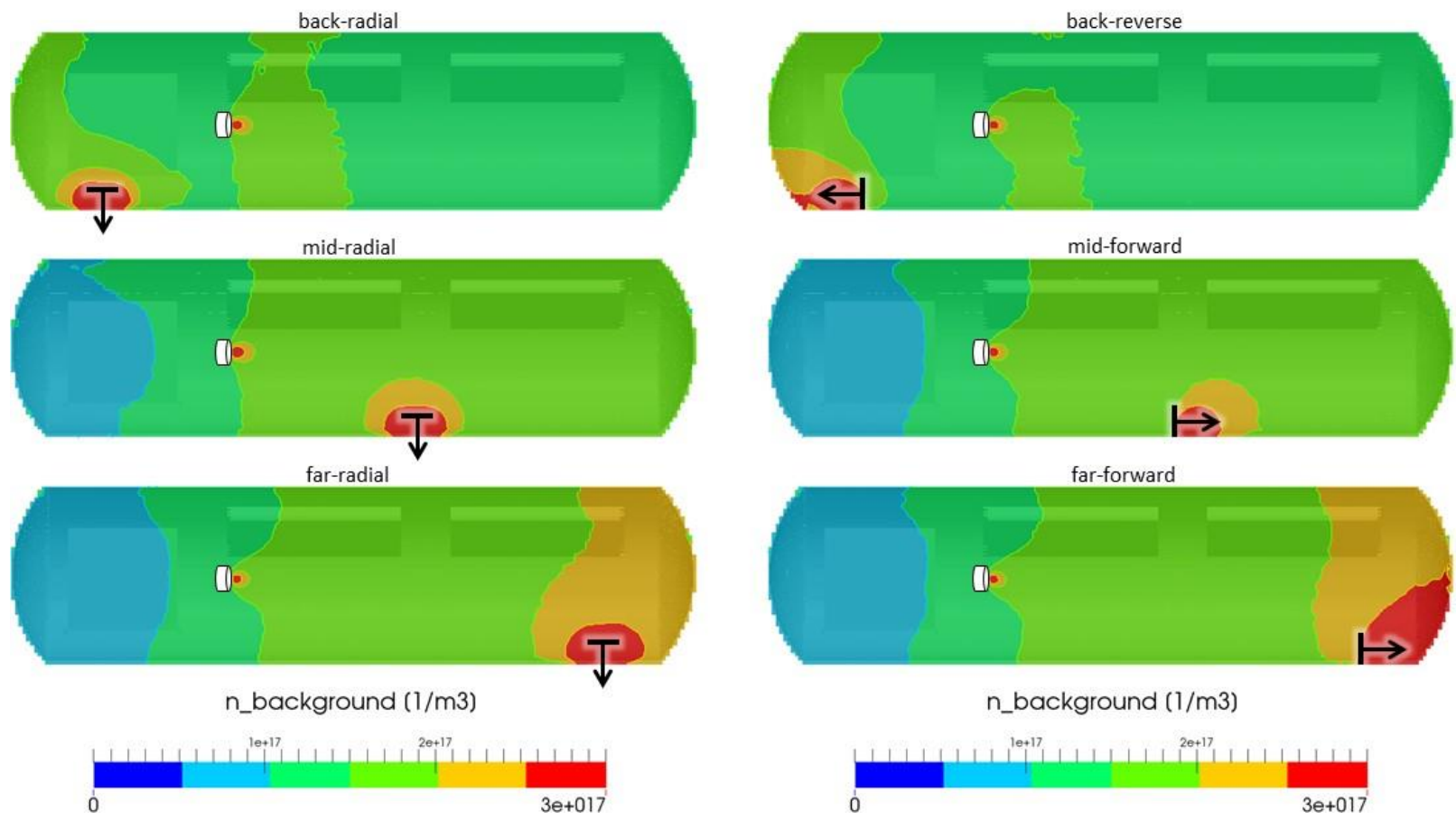

Figure 10: The background gas number density profiles for six different supplemental gas inflow configurations.

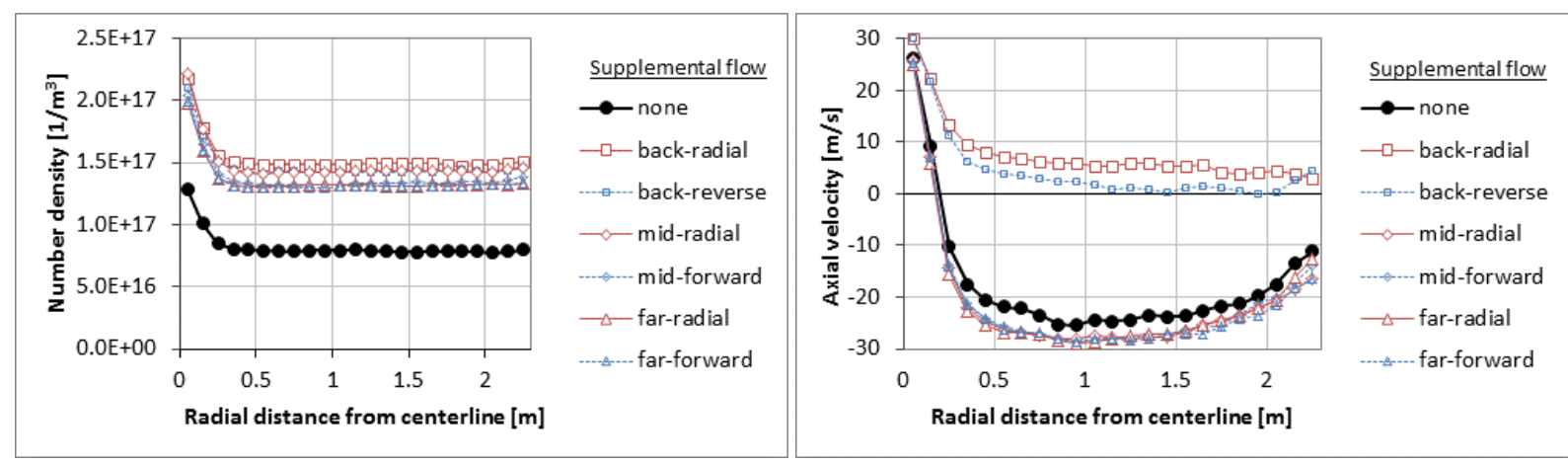

Figure 11: The radial variation of the number density (left) and axial velocity (right) at the thruster exit plane for various supplemental gas inflow configurations.

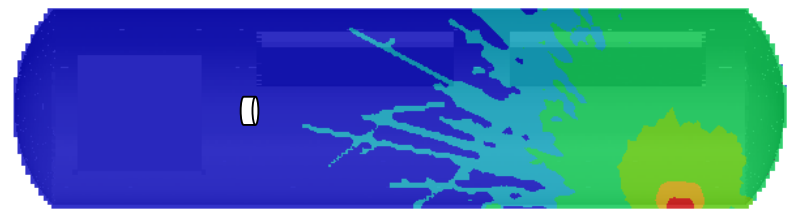

n_added $(1 / \mathrm{m} 3)$

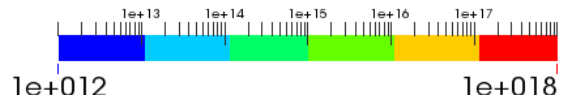

10+018

Figure 12: The unimpeded supplemental gas density profiles for two different inflow locations.

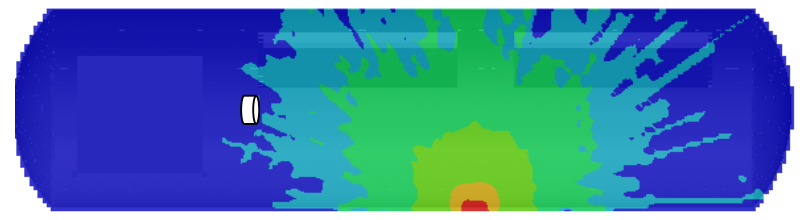

n_added $(1 / \mathrm{m} 3)$

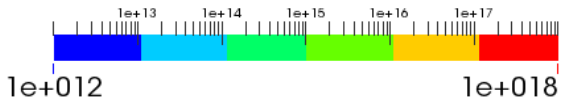




\section{Implications for flux calculations}

Typically, the background gas flux at the thruster has been calculated using the classical solution for molecular efflux in one direction given by

$$
\Phi_{0}=n \sqrt{\frac{k T}{2 \pi m}}=\frac{1}{4} n v_{t h}
$$

This equation is derived under the assumption that the gas is at rest with no bulk velocity. However, as shown above, there is a clear bulk velocity for the vacuum facility background gas. If we introduce a bulk velocity, $v_{B}$, into the particle velocity distribution, the corresponding particle flux in the direction of the bulk velocity can be evaluated to be

$$
\begin{aligned}
\Phi_{B} & =n \sqrt{\frac{m}{2 \pi k T}} \int_{0}^{\infty} v_{x} e^{-\frac{m}{2 k T}\left(v_{x}-v_{B}\right)^{2}} d v_{x} \sqrt{\frac{m}{2 \pi k T}} \int_{-\infty}^{\infty} e^{-\frac{m}{2 k T} v_{y}^{2}} d v_{y} \sqrt{\frac{m}{2 \pi k T}} \int_{-\infty}^{\infty} e^{-\frac{m}{2 k T} v_{z}^{2}} d v_{z} \\
& =\frac{1}{4} n v_{t h}\left(e^{-\left(\frac{2}{\sqrt{\pi}} \frac{v_{B}}{v_{t h}}\right)^{2}}+2 \frac{v_{B}}{v_{t h}}\left[1+\operatorname{erf}\left(\frac{2}{\sqrt{\pi}} \frac{v_{B}}{v_{t h}}\right)\right]\right)
\end{aligned}
$$

It is then straightforward to calculate the ratio of the flux for a gas with a bulk velocity to a gas without a bulk velocity, and this is shown in Figure 13. Note for these results, a negative bulk velocity indicates flow moving upstream relative to the thruster, whereas a positive bulk velocity is moving in the same direction as the flow exiting the thruster. For the specific facility and thruster example shown above, where the bulk velocity is around $20 \mathrm{~m} / \mathrm{s}$ in the upstream direction and the thermal velocity of xenon gas at room temperature is roughly $200 \mathrm{~m} / \mathrm{s}$, the potential ingestion flux is then expected to be approximately $20 \%$ higher than a static background gas with no bulk velocity. Sample DSMC vacuum facility simulation data points are also provided, showing general good agreement with the free molecular flow theory for this particular case.

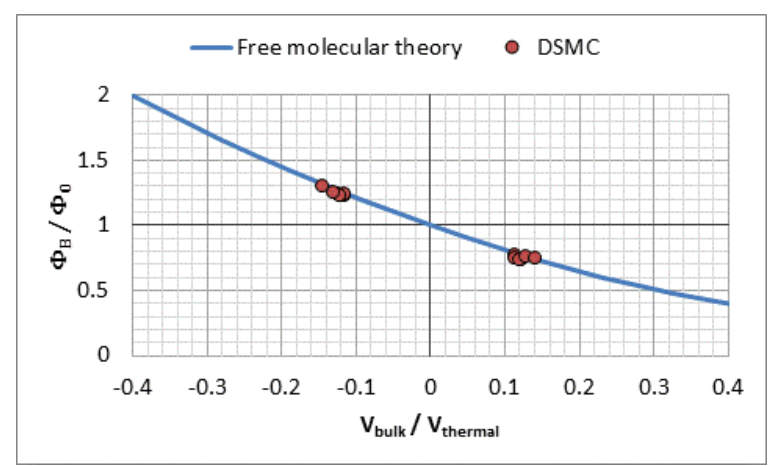

Figure 13: The ratio of number flux from free molecular flow with bulk velocity-and sample DSMC results-to the classical flux for a gas at rest as a function of the ratio of the bulk velocity to the gas thermal velocity.

It is important to note that both Eq. (2) and Eq. (3) are derived assuming the gas is in equilibrium, with a Maxwellian distribution. The assumption of gas equilibrium should not be taken for granted under vacuum facility conditions. Expanding flows, as from the thruster into the relative vacuum of the facility, are a well known example of non-equilibrium flow. ${ }^{24}$ In addition, the compounding factors of the near free molecular regime of vacuum facility flow $(\mathrm{Kn}>1)$ and surfaces at different temperatures including a hot thruster, room temperature facility walls, and cold cryopumps further contribute to the likelihood of non-equilibrium nature for the background gas.

The axial (here denoted as the ' $\mathrm{X}$ ' direction) and the two lateral components (denoted ' $\mathrm{Y}$ ' and ' $\mathrm{Z}$ ') of the background gas VDFs are examined separately, as shown in the left side of Figure 14. While the $\mathrm{Y}$ and $\mathrm{Z}$ components of the velocity appear to be similar to each other, the axial component is clearly shifted relative to the other two, indicating a non-equilibrium flow. This is further seen when the corresponding temperatures and pressure tensors are calculated. The right side of Figure 14 shows that the component in the axial direction is clearly not equivalent to the two lateral components at all radial distances along the thruster exit plane. These results indicate that the overall velocity distribution is anisotropic as well as drifting. 

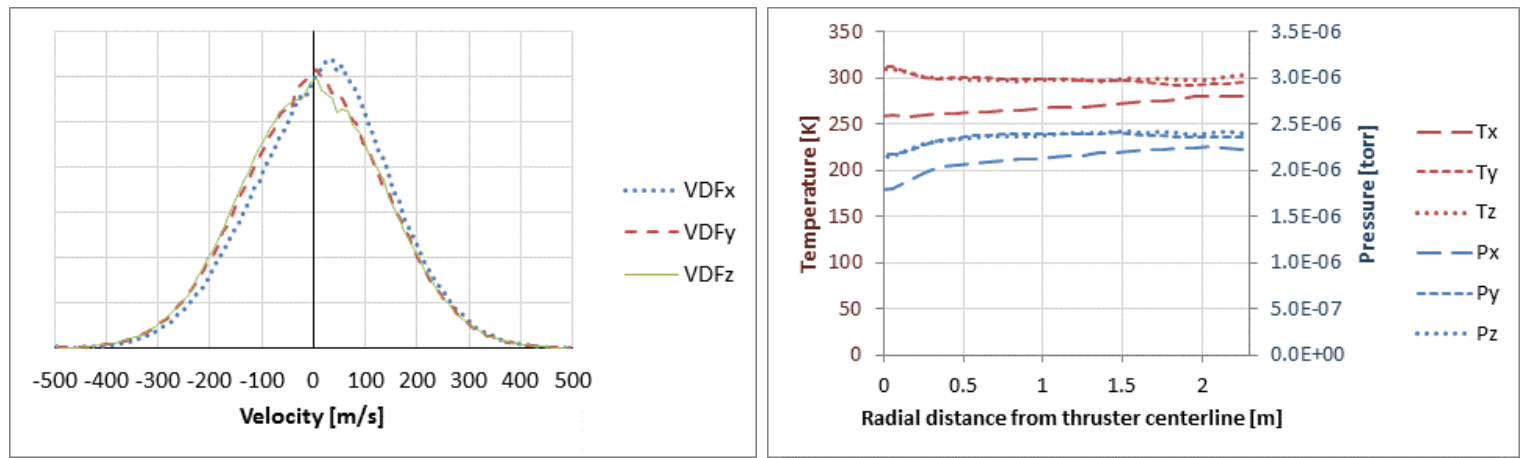

Figure 14: Sample component direction velocity distribution functions (left) and temperatures and pressure tensor components calculated from the three velocity component directions (right) along the thruster exit plane.

Along these specified component directions, however, the individual VDFs still appear to maintain a Gaussian form. Sample goodness-of-fit tests such as evaluating skewness or using the Kolmogorov-Smirnov (K-S) test were applied to assess the component VDFs. Skewness was found to be between -0.15 and -0.10 for the VDFs examined, well above commonly applied thresholds of -0.7 or -1.0 . A sample evaluated K-S statistic was found to be 0.022 , which fits below the threshold of 0.025 required to meet a significance level of 0.01 that the null hypothesis that the VDFs are a Maxwellian distribution is not rejected. The results, then, are in somewhat fair approximation to a Maxwellian distribution, though clearly not perfect. If these specific individual components of the velocity are then able to be represented by a Maxwellian distribution but with different components of translational kinetic temperature, then the flux calculated from the corresponding velocity distribution functions can still be approximated by Eq. (3).

\section{Conclusions}

Vacuum facilities used in ground testing of electric propulsion devices are found to have a relatively low, but finite, background gas flow velocity. The presence of this bulk velocity has a few effects that should be considered when configuring tests and evaluating data. Depending on the configuration of the facility and the location and orientation of the thruster, the flux of the background gas ingested by the thruster may be significantly different than expected using the classical flux calculation for a static gas. The presence of a bulk velocity in the background gas may also affect its interaction and ingestion by the thruster, though those details are outside the scope of this work. Use of supplemental gas flows to artificially increase the back pressure of a facility should be carefully planned to not significantly change the nature of the ingested flux at the thruster.

Characterization of the background pressure should be standardized where possible. The results here suggest measurements located radially away from the thruster but along the thruster exit plane may provide the best measurement of just the background gas properties ingested by the thruster. Care should be taken to avoid locating the pressure gauge near facility pumps, and the gauge should also avoid being placed near objects that may perturb the local flow or induce conductance losses. The pressure measurement may also be significantly different than the actual local pressure within the facility depending on the orientation of the neutralizer tube. A neutralizer tube inlet that is oriented perpendicular to the direction of expected background gas flow should provide the best measurement of the local static pressure. Additional gauges are recommended pointing into or away from the flow to allow calculations of the background gas flow velocity. It should again be noted that the quantitative conclusions presented in this paper cannot be generalized to all vacuum facility configurations, and care should be taken for each specific setup to assess or estimate the behavior of the background gas and its effect on measurements and thruster operation.

Future work to further analyze the properties of the background gas could include examination of a hot fire case to assess the influence of the diffuse cold inflow on these results, particularly in regions close to the thruster. Of course, inclusion of a full plasma plume with charge exchange collisions would help to further quantify the effect of the background gas on thruster operation and performance. The inclusion of more complex geometry, especially the thrust stand and support structure, would also impact the near thruster flow dynamics as they would create regions of localized flow stagnation and pressure variations. Finally, expanding this work across different facilities and thruster configurations would help to characterize the extent of differences in the background gas properties across various test setups. 


\title{
Acknowledgments
}

The first author would like to thank the support of NASA's Space Technology Mission Directorate's Solar Electric Propulsion Technology Demonstration Mission project and all of the team members who aided in the collection and interpretation of facility pressure data. The authors would also like to thank Hani Kamhawi and Wensheng Huang of NASA Glenn Research Center for the data from HiVHAc thruster testing.

\section{References}

\begin{abstract}
${ }^{1}$ Randolph, T., et al., "Facility Effects on Stationary Plasma Thruster Testing," $23^{\text {rd }}$ International Electric Propulsion Conference, IEPC 93-93, 1993.

${ }^{2}$ Byers, D. and Dankanich, J. W., "A Review of Facility Effects on Hall Effect Thrusters," $31^{\text {st }}$ International Electric Propulsion Conference, IEPC-2009-076, 2009.

${ }^{3}$ Nakles, M. R. and Hargus Jr., W. A., "Background Pressure Effects on Ion Velocity Distribution Within a Medium-Power Hall Thruster," Journal of Propulsion and Power, Vol. 27, No. 4, pp. 737-743, 2011.

${ }^{4}$ Mazouffre, S., Bourgeois, G. Garrigues, L, and Pawelec, E., "A Comprehensive Study on the Atom Flow in the Cross-Field Discharge of a Hall Thruster," Journal of Physics D: Applied Physics, Vol. 44, 105203, 2011.

${ }^{5}$ Diamant, K. D., Spektor, R., Beiting, E. J., Young, J. A., and Curtiss, T. J., "The Effects of Background Pressure on Hall Thruster Operation," 48 th AIAA/ASME/SAE/ASEE Joint Propulsion Conference and Exhibit, AIAA 2012-3735, 2012.

${ }^{6}$ Boyd, I. D. and Crofton, M. W., "Numerical Momentum Tracking for a Hall Thruster Plume," 50 ${ }^{\text {th }}$ AIAA/ASME/SAE/ASEE Joint Propulsion Conference, AIAA-2014-3428, 2014.

${ }^{7}$ Kamhawi, H., Huang, W., Haag, T., and Spektor, R., "Investigation of the Effects of Facility Background Pressure on the Performance and Voltage-Current Characteristics of the High Voltage Hall Accelerator," 50 ${ }^{\text {th }}$ AIAA/ASME/SAE/ASEE Joint Propulsion Conference, AIAA-2014-3707, 2014.

${ }^{8}$ Huang, W., Kamhawi, H., Lobbia, R. B., and Brown, D. L., "Effect of Background Pressure on the Plasma Oscillation Characteristics of the HiVHAc Hall Thruster," 50 th AIAA/ASME/SAE/ASEE Joint Propulsion Conference, AIAA-2014-3708,
\end{abstract} 2014.

${ }^{9}$ Hofer, R. R. and Anderson, J. A., "Finite Pressure Effects in Magnetically Shielded Hall Thrusters," 50 ${ }^{\text {th }}$ AIAA/ASME/SAE/ASEE Joint Propulsion Conference, AIAA-2014-3709, 2014.

${ }^{10}$ Diamant, K. D., Liang, R., and Corey, R. L., "The Effect of Background Pressure on SPT-100 Hall Thruster Performance," 50 $0^{\text {th }}$ AIAA/ASME/SAE/ASEE Joint Propulsion Conference, AIAA-2014-3710, 2014.

${ }^{11}$ Dankanich, J. W., Walker, M., Swiatek, M. W., and Yim, J. T., "Recommended Practice for Pressure Measurements and Calculation of Effective Pumping Speeds During Electric Propulsion Testing," 33rd International Electric Propulsion Conference, IEPC-2013-358, 2013.

${ }^{12}$ Kamhawi, H., et al., "Overview of the Development of the Solar Electric Propulsion Technology Demonstration Mission 12.5-kW Hall Thruster," 50 th AIAA/ASME/SAE/ASEE Joint Propulsion Conference, AIAA-2014-3898, 2014.

${ }^{13}$ Burt, J. M., Josyula, E., and Boyd, I. D., "Novel Cartesian Implementation of the Direct Simulation Monte Carlo Method," Journal of Thermophysics and Heat Transfer, Vol. 26, No. 2, pp. 258-270, 2012.

${ }^{14}$ Walker, M. L. R., Gallimore, A. D., Boyd, I. D., and Cai, C., "Vacuum Chamber Pressure Maps of a Hall Thruster ColdFlow Expansion," Journal of Propulsion and Power, Vol. 20, No. 6, pp. 1127-1131, 2004.

${ }^{15}$ Yim, J. T., Herman, D. A., and Burt, J. M., "Modeling Analysis for NASA GRC Vacuum Facility 5 Upgrade," NASA/TM-2013-216496, 2013.

${ }^{16}$ Crofton, M. W., et al., "Neutral Xenon Density in the SPT-140 Near-Field Plume," 33 ${ }^{\text {rd }}$ International Electric Propulsion Conference, IEPC-2013-399, 2013.

${ }^{17}$ Boyd, I. D., Sun, Q., and Cai, C., "Particle Simulation of Hall Thruster Plumes in the 12V Vacuum Chamber," $29^{\text {th }}$ International Electric Propulsion Conference, IEPC-2005-138, 2005.

${ }^{18}$ Hughes, P. C. and de Leeuw, J. H., "Theory for the Free Molecule Impact Probe at an Angle of Attack," Rarefied Gas Dynamics: Proceedings of the Fourth International Symposium on Rarefied Gas Dynamics, Academic Press, Vol. 1, pp. 653-676, 1965.

${ }^{19}$ de Leeuw, J. H. and Rothe, D. E., "A Numerical Solution for the Free-Molecule Impact Pressure Probe Relations for Tubes of Arbitrary Length," UTIA report, No. 88, 1962.

${ }^{20}$ Koshmarov, Y. A. and Svirshchevskii, S. V., "Interaction Between a Total-Pressure Tube and a Subsonic Flow of Rarefied Gas," Fluid Dynamics, Vol. 6, No. 2, pp. 694-697, 1971.

${ }^{21}$ Kleber, P., "Pressure, What Does It Mean in Vacuum Chambers with Cryosurfaces?" Vacuum, Vol. 25, No. 5, pp. 191-196, 1975.

${ }^{22}$ Haefer, R. A., "The Application of Ionization Vacuum Gauges to Measurements in Vacuum Chambers Provided with CryoPumping Surfaces" Vacuum, Vol. 30, No. 4/5, pp. 193-195, 1980.

${ }^{23}$ Kamhawi, H., et al., "Performance Characterization of the Solar Electric Propulsion Technology Demonstration Mission 12.5-kW Hall Thruster" 34 ${ }^{\text {th }}$ International Electric Propulsion Conference, IEPC-2015-07, 2015.

${ }^{24}$ Bird, G. A., "Breakdown of Translational and Rotational Equilibrium in Gaseous Expansions," AIAA Journal, Vol. 8, No. 11, pp. 1998-2003, 1970. 